\title{
Manifestações clínicas e desafios diagnósticos na Síndrome de incontinentia pigmenti
}

\section{Multiple clinical manifestations and diagnostic challenges of incontinentia pigmenti Syndrome}

Paula Kataguiri ${ }^{1}$, Felipe Cortizas Ré Martins², Vivien Yamada ${ }^{3}$,Gustavo Salomão ${ }^{4}$, Rodrigo Ribeiro ${ }^{5}$, José Ricardo Carvalho Lima Rehder ${ }^{6}$

\section{$\underline{\text { ResUMO }}$}

A Síndrome de Incontinentia Pigmenti (Síndrome de Bloch-Sulzberger) é uma doença rara, ligada ao cromossomo X e envolve tecidos ectodérmicos de múltiplos órgãos. As manifestações oculares surgem ao nascimento ou após algumas semanas. O objetivo desse trabalho é relatar as características oftalmológicas, dermatológicas e os achados radiológicos de uma paciente com a Síndrome de Incontinentia Pigmenti. Trata-se de uma paciente do sexo feminino, 26 anos que procurou o serviço de Oftalmologia da Faculdade de Medicina do ABC com queixa de baixa de visão em ambos os olhos há 1 ano. Apresentava alterações retinianas importantes e lesões cutâneas e dentárias sugestivas de síndrome de Incontinentia Pigmenti, confirmada por biópsia de pele. Conclui-se que o diagnóstico precoce é essencial, pois as lesões retinianas podem levar a sequelas graves e cegueira.

Descritores: Incontinência pigmentar; Síndrome de Bloch-Sulzberger; Manifestações oculares; Biópsia; Relatos de casos

\footnotetext{
${ }^{1}$ Residente do Segundo Ano de Oftalmologia da Faculdade de Medicina do ABC - FMABC - Santo André (SP), Brasil; ${ }^{2}$ Residente do Terceiro Ano de Oftalmologia da Faculdade de Medicina do ABC - FMABC - Santo André (SP), Brasil; ${ }^{3}$ Estagiária de Dermatologia da Faculdade de Medicina do ABC - FMABC - Santo André (SP), Brasil; ${ }^{4}$ Médico Colaborador do Setor de Oftalmopediatria da Faculdade de Medicina do ABC - FMABC - Santo André (SP), Brasil; ${ }^{5}$ Médico Colaborador do Setor de Retina da Faculdade de Medicina do ABC - FMABC - Santo André (SP), Brasil; ${ }^{6}$ Professor Titular da Disciplina de Oftalmologia da Faculdade de Medicina do ABC - FMABC - Santo André (SP), Brasil.

Trabalho realizado no setor de Oftalmologia da Faculdade de Medicina do ABC - FMABC - Santo André (SP), Brasil.
} 


\section{INTRODUÇÃO}

I ncontinentia Pigmenti ou Síndrome de BlochSulzberger é uma doença rara, ligada ao cromossomo X, de caráter autossômico dominante e geralmente, letal no sexo masculino. Essa síndrome afeta tecidos ectodérmicos, como os dentes, ossos, olhos e sistema nervoso central ${ }^{(1)}$. A manifestação clínica mais evidente é a alteração dermatológica que se divide em 4 estágios: se inicia ao nascimento com eritema e lesões bolhosas, evoluindo para lesões verrucosas e, posteriormente para áreas de hiperpigmentação disseminadas e irregulares; o quarto estágio visto em adultas, se caracteriza por lesões hipocrômicas e atróficas. A histopatologia mostra um aumento de eosinófilos nos primeiros estágios, e posteriormente, depósitos de melanina. O diagnóstico é feito através da combinação entre as características físicas, biópsia de pele e história familiar positiva ${ }^{(2)}$.

Manifestações dentárias ocorrem em $65 \%$ dos casos, e inclui anodontia, dentição atrasada e dentes malformados. Porém, os distúrbios visuais e neurológicos são os aspectos mais significantes na doença ${ }^{(3)}$.

Manifestações oculares, geralmente assimétricas, são descritas em $35 \%$ dos casos. As principais características incluem lesões retinianas isquêmicas e neovascularização com subsequente hemorragia e exsudação, podendo ocorrer descolamento retiniano tracional. Assim como a retinopatia da prematuridade, $o$ processo pode estacionar em qualquer estágio, levando a diversas sequelas: áreas avasculares, vasos tortuosos e irregulares, exsudatos, hemorragia vítrea, fibrose préretiniana, alteração do epitélio pigmentar da retina e descolamento da retina. Além das alterações retinianas, foram observados atrofia óptica, hipoplasia foveal,

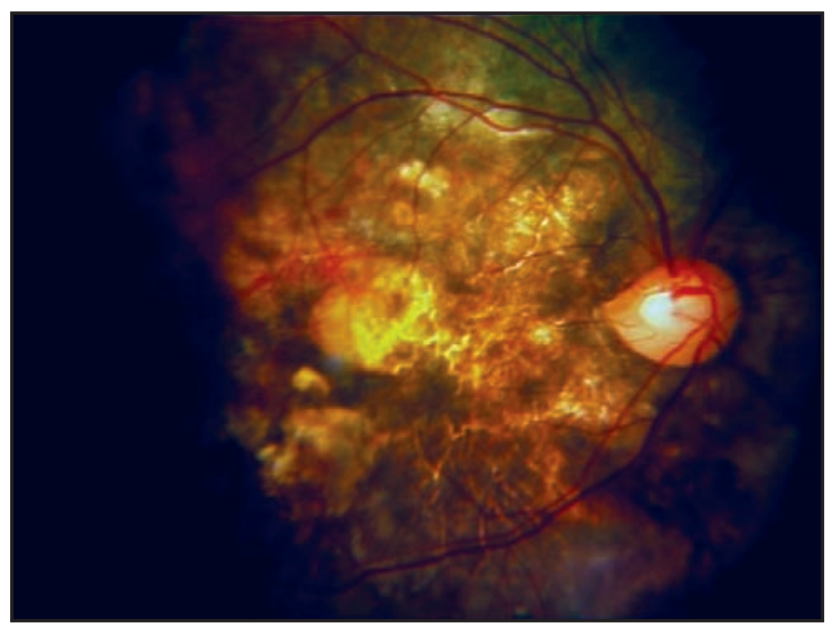

Figura 2: Retinografia revelando áreas de hipopigmentação com focos de hiperplasia do epitélio pigmentado da retina em ambos olhos
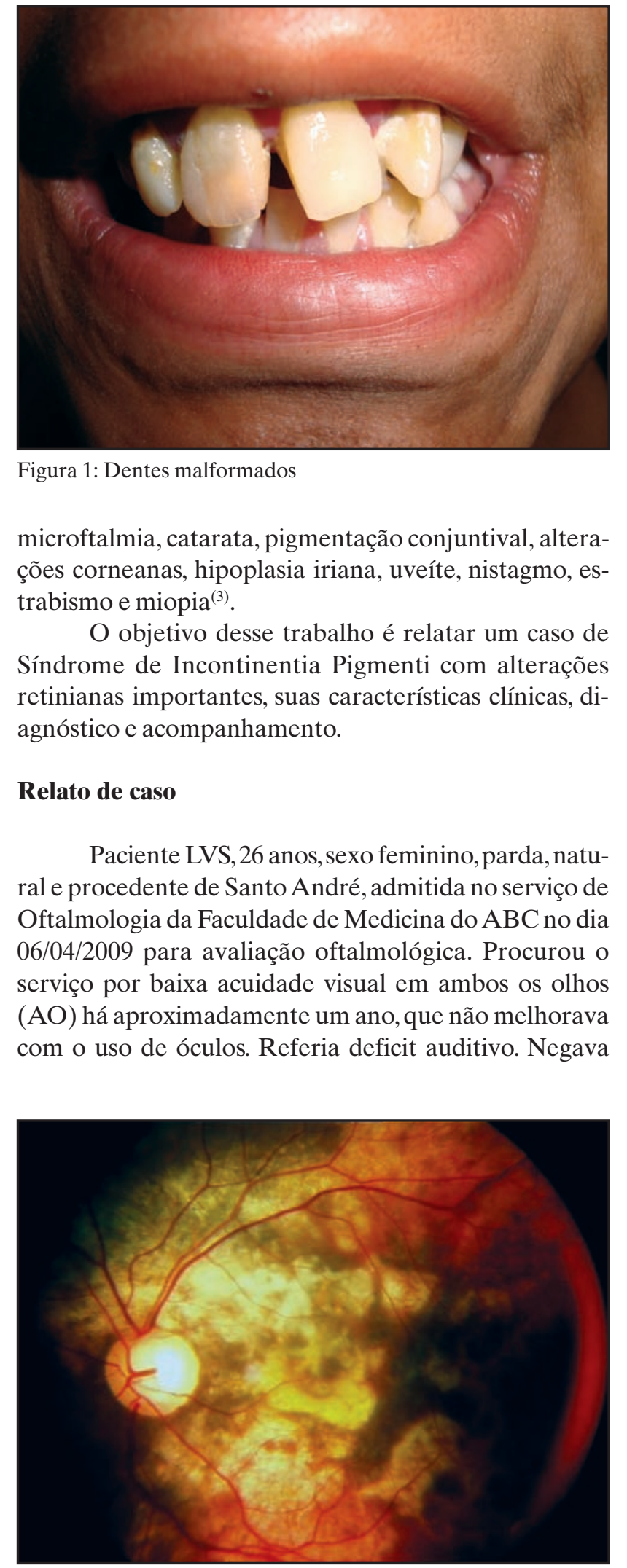

Figura 1: Dentes malformados

microftalmia, catarata, pigmentação conjuntival, alterações corneanas, hipoplasia iriana, uveíte, nistagmo, estrabismo e miopia ${ }^{(3)}$.

O objetivo desse trabalho é relatar um caso de Síndrome de Incontinentia Pigmenti com alterações retinianas importantes, suas características clínicas, diagnóstico e acompanhamento.

\section{Relato de caso}

Paciente LVS, 26 anos, sexo feminino, parda, natural e procedente de Santo André, admitida no serviço de Oftalmologia da Faculdade de Medicina do ABC no dia 06/04/2009 para avaliação oftalmológica. Procurou o serviço por baixa acuidade visual em ambos os olhos (AO) há aproximadamente um ano, que não melhorava com o uso de óculos. Referia deficit auditivo. Negava 

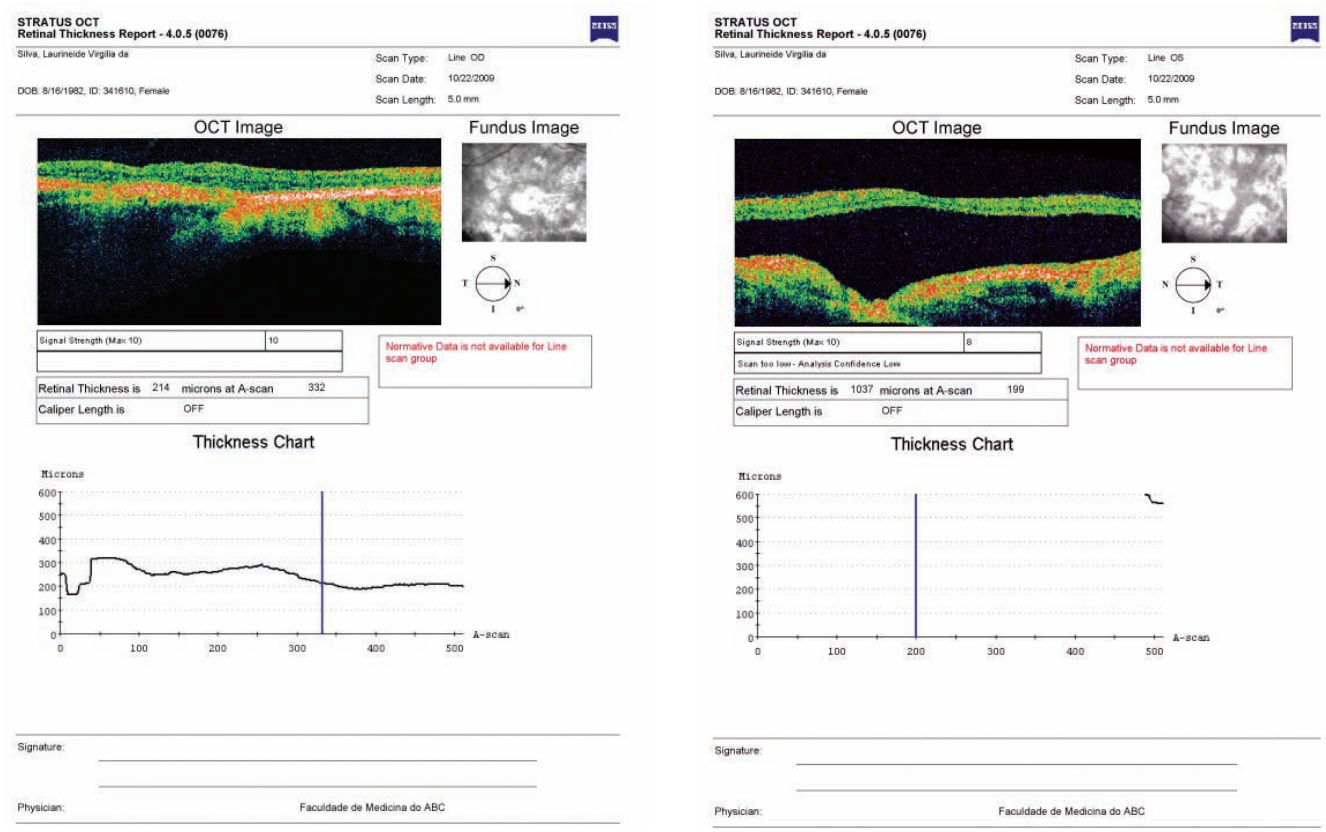

Figura 3: Áreas de desorganização do epitélio pigmentado da retina em olho direito e descolamento neurossensorial da retina em olho esquerdo

antecedentes familiares ou consanguinidade. Negava alterações cardíacas, pulmonares ou renais. Ao exame externo apresentava alopécia e alterações dentárias (Figura 1). Ao exame oftalmológico, a acuidade visual corrigida era de 20/100 no olho direito (OD) e 20/100 no olho esquerdo (OE). A refração no OD -3,00DE $0,25 \mathrm{DC} \times 150^{\circ}$ e no OE -0,75DE; a biomicroscopia do segmento anterior não mostrou alterações $\mathrm{AO}$; a pressão intraocular foi de $14 \mathrm{mmHg}$ em $\mathrm{AO}$; paquimetria OD: 475 e OE: 485; microscopia especular OD: 2615 células e OE: 2180 células e à fundoscopia observou-se áreas de hipopigmentação com focos de hiperpigmentação do epitélio pigmentado da retina em ambos olhos (Figura 2).

Foram realizadas ainda tomografia de coerência óptica (OCT) e angiofluoresceínografia. O OCT demonstrou hiperrefletividade e áreas de desorganização do epitélio pigmentado da retina em olho direito e descolamento neurossensorial em olho esquerdo (Figura 3). A angiografia fluoresceínica revelou áreas de hipofluorescência por bloqueio entremeadas por áreas de hiperfluorescência transmitida (defeito em janela) em ambos os olhos, além de ausência da zona avascular da fóvea em olho direito (Figura 4).

Posteriormente, acompanhada da mãe, que nos informa que a paciente realiza acompanhamento em outro serviço (AACD) por deficit cognitivo, e traz laudo médico e exames, os quais comprovam a presença de escoliose tóracolombar, onde foi submetida à artrodese de coluna vertebral em agosto de 1999 (Figura 5).

Ao exame dermatológico apresentava máculas hipercrômicas bem delimitadas e bordas irregulares em virilha, região axilar, torácica e abdominal (Figura 6).

Os exames oculares somados às alterações sistêmicas confirmam que a paciente apresenta a Síndrome de Incontinentia Pigmenti (Síndrome de BlochSulzberger). Solicitado biópsia de pele para confirmação diagnóstica, cujo resultado corresponde à terceira fase da Síndrome de Incontinentia Pigmenti. Ao exame macroscópico: fragmento discóide de pele que mede 0,2 $\mathrm{x} 0,2 \mathrm{~cm}$, de cor enegrecida e consistência firme-elástica (material fixado em formalina). Ao exame microscópico: a histologia revela fragmento de pele que apresenta epiderme de espessura irregular. Observa-se discreta fibroplasia da derme e melanófagos ao redor dos vasos. Conclusão: discreto derrame pigmentar (Figura 7).

\section{DısCUSSÃO}

Embora o rash cutâneo característico na Síndrome de Incontinentia Pigmenti seja o fator mais evidente para os médicos e pais, o caso relatado demonstra que as anormalidades neurológicas e oftalmológicas são as que geram maior morbidade para o paciente. 

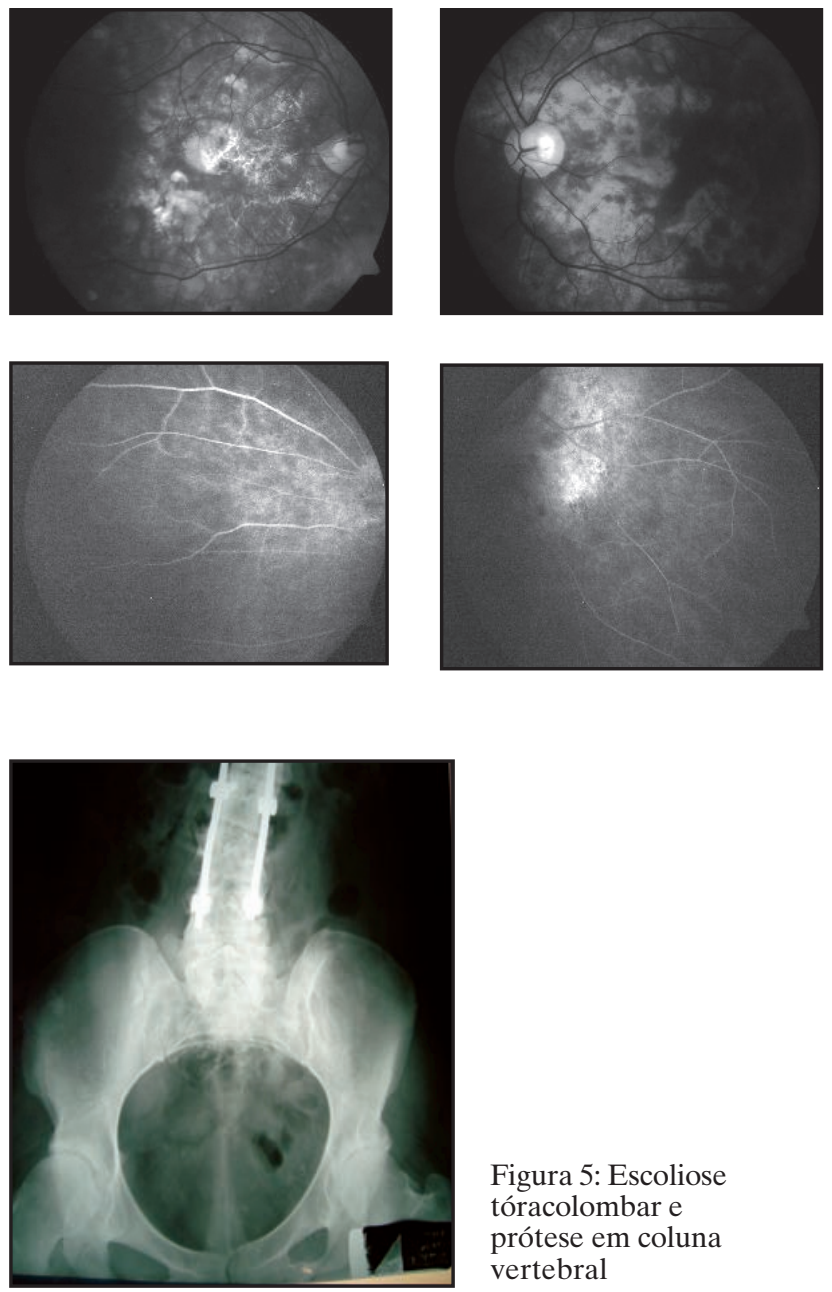

Figura 5: Escoliose tóracolombar e prótese em coluna vertebral

Uma vez que a doença é uma entidade rara, não impressiona o fato de ser uma patologia subdiagnosticada, a qual pouco se conhece a patogenia e história natural. Segundo Nishimura et al. ${ }^{(5)}$, ocorre uma alteração no epitélio pigmentar da retina, levando a displasia e descolamento na retina, semelhante ao caso relatado. Além disso, insuficiência arterial primária gera obstrução, isquemia e proliferação anormal dos vasos. Por outro lado, Brown defende que a isquemia retiniana pode ser resultado de um fator angiogênico ${ }^{(6)}$. Já Catalano et al. descrevem alterações enzimáticas e inflamatórias, as quais estimulam uma proliferação fibrovascular anormal e consequente displasia retiniana ${ }^{(7)}$.

A história natural das lesões retinianas não é totalmente compreendida. As alterações vasculares parecem ter início nas primeiras semanas de vida e progridem após o nascimento por semanas ou meses. Esse processo pode estabilizar em qualquer estágio, deixando inúmeras sequelas ${ }^{(5,8)}$. Para Morton Goldberg, a isquemia macular progressiva pode ocorrer na síndrome, e seria o
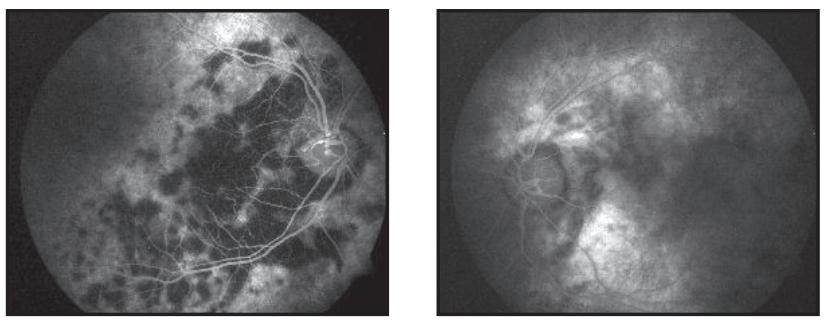

Figura 4.

Angiofluoreceinografia evidenciando áreas de hiperfluorescência por defeito em janela entremeadas por áreas de hipofluorescência por bloqueio em ambos os olhos
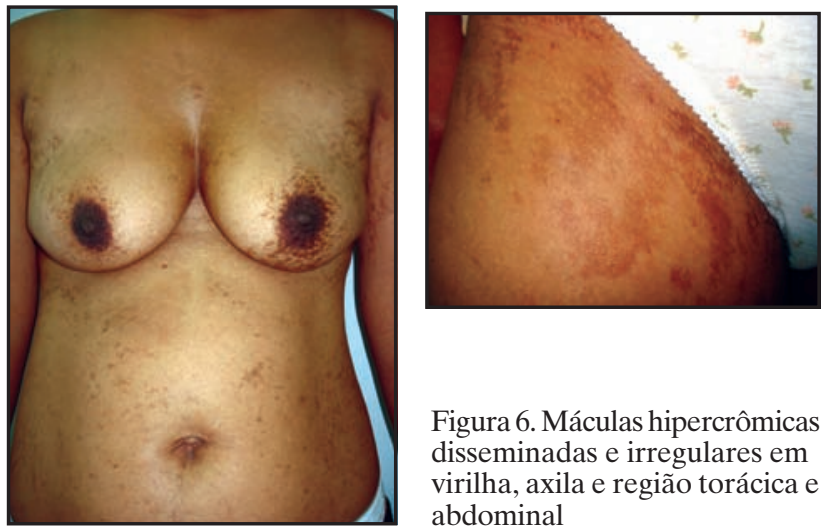

Figura 6. Máculas hipercrômicas disseminadas e irregulares em virilha, axila e região torácica e abdominal

início da vasculopatia típica da doença com consequente neovascularização e descolamento tracional da retina. É possível que a eosinofilia na fase inicial da doença possa contribuir para o aumento de viscosidade nos leitos capilares perifoveais e periféricos da retina, assim como nos vasos sanguíneos cerebrais, e resulta na obstrução vascular ${ }^{(9)}$.

A angiofluoresceinografia permite o diagnóstico e determinação do local da isquemia retiniana. Os sinais angiográficos representam uma alteração típica no epitélio pigmentar da retina e difusão do corante ao nível das anastomoses artériovenosas na região foveal associada à hipoplasia foveal ${ }^{(10)}$. O caso relatado é compatível com a literatura, uma vez que é possível observar na angiofluoresceínografia ausência de zona avascular da fóvea.

Os tratamentos para a Síndrome de Incontinentia Pigmenti também são restritos. Crio ou fotocoagulação a laser são recomendados, quando os sinais de lesão retiniana são vistos no início da doença. Tratamentos das áreas avasculares ou isquêmicas representam as 

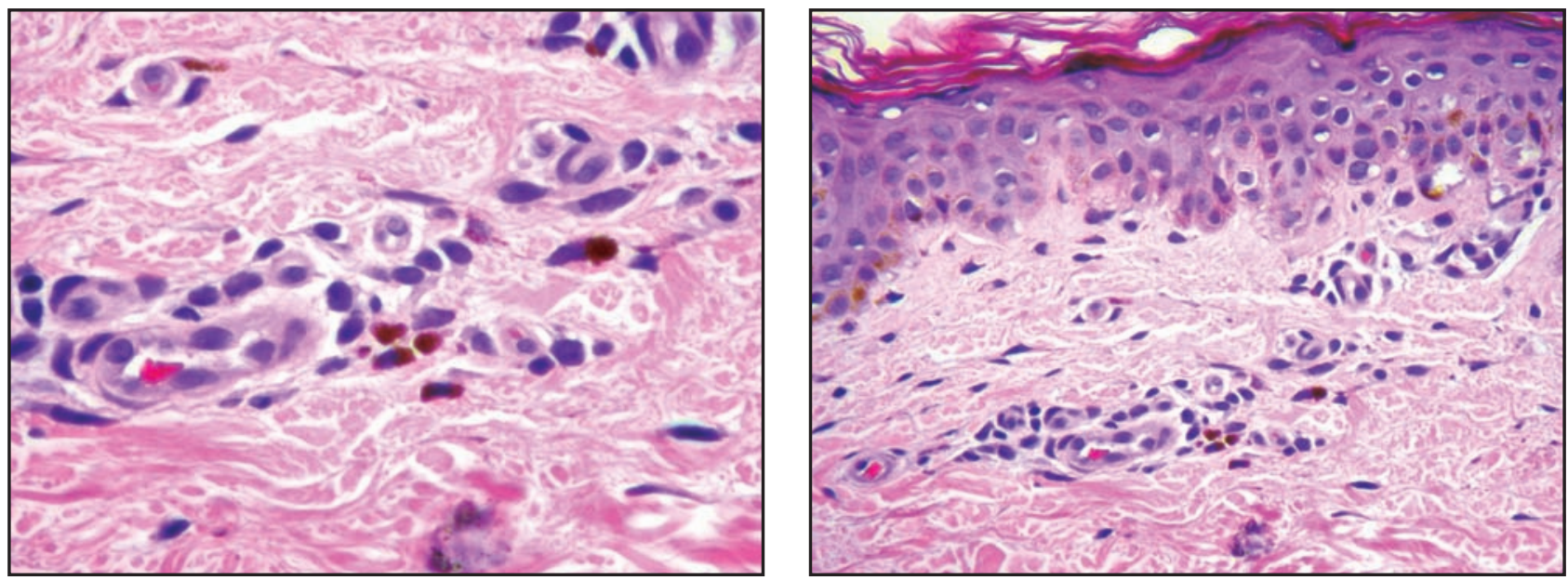

Figura 7: Histologia revela fragmento de pele que apresenta epiderme de espessura irregular. Observa-se discreta fibroplasia da derme e melanófagos ao redor dos vasos

opções mais comuns, porém com resultados variáveis. Em um pequeno número de casos avançados de descolamento de retina tracional, foi realizado vitrectomia, mas sem sucesso.

\section{Conclusão}

As lesões oculares na Síndrome de Incontinentia Pigmenti podem levar à cegueira, pois os eventos isquêmicos ocorrem logo após o nascimento. Portanto,é necessário o reconhecimento precoce dessa patologia e acompanhamento multidisciplinar.

\footnotetext{
Abstract

Incontinentia Pigmenti (Bloch-Sulzberger syndrome) is a rare, $X$-linked dominant disorder that involves ectodermal tissues of multiple systems. Cutaneous manifestations appear at birth or within a few weeks after. We aimed to describe ophthalmological, dermatological and radio diagnostic findings of a patient with Incontinentia Pigmenti. A twenty-six year old girl was followed at ABC Medicine School for visual loss, retina and cutaneous lesions with the diagnosis of Incontinentia Pigmenti confirmed by a skin biopsy. She had delayed eruption of her teeth some of which were conical. To conclude early management of retinal changes in Incontinentia Pigmenti is essential because they may be serious leading to blindness.

Keywords: Incontinentia Pigmenti; BlochSulzberger syndrome; Eye manifestations; Biopsy; Case reports
}

\section{RefERÊNCIAS}

1. Chang JT, Chiu PC, Chen YY, Wang HP, Hsieh KS. Multiple clinical manifestations and diagnostic challenges of incontinentia pigmenti--12 years' experience in 1 medical center. J Chin Med Assoc. 2008;71(9):455-60.

2. Lever W. Lever's histopathology of the skin. 8th ed. Philadelphia: Lippincott-Raven; c1997.

3. Holmström G, Thorén K. Ocular manifestations of incontinentia pigmenti. Acta Ophthalmol Scand. 2000;78(3):348-53.

4. Carney RG. Incontinentia pigmenti. A world statistical analysis. Arch Dermatol. 1976;112(4):535-42.

5. Nishimura M, Oka Y, Takagi I, Yamana T, Kitano A. The clinical features and treatment of the retinopathy of BlochSulzberger syndrome (incontinentia pigmenti). Jpn J Ophthalmol. 1980;24:310-9.

6. Brown CA. Incontinentia pigmenti: the development of a pseudoglioma. Br J Ophthalmol. 1988;72(6):452-5.

7. Catalano RA, Lopatysnky M, Tasman WS. Treatment of proliferative retinopathy associated with incontinentia pigmenti. Am J Ophthalmol. 1990;110(6):701-2.

8. Goldberg MF, Custis PH. Retinal and other manifestations of incontinentia pigmenti (Bloch-Sulzberger syndrome). Ophthalmology. 1993;100(11):1645-54.

9. Goldberg MF. Macular vasculopathy and its evolution in incontinentia pigmenti. Ophthalmic Genet. 1998;19(3):141-8.

10. Tnacheri Ouazzani B, Guedira K, Dali H, Laghmari M, Ibrahimy W, Daoudi R, et al. Incontinentia pigmenti: à propos d'un cas. J Fr Ophtalmol. 2007;30(8):e24.

\section{Endereço para correspondência: \\ Paula Kataguiri}

Rua Fradique Coutinho, $n^{\circ} 546$ apto. 181 - Pinheiros

CEP 05416-000 - São Paulo (SP), Brasil

paula_kataguiri@yahoo.com.br 